Pacific Journal of Mathematics

RICCATI MATRIX DIFFERENTIAL EQUATIONS AND
NON-OSCILLATION CRITERIA FOR ASSOCIATED LINEAR
DIFFERENTIAL SYSTEMS

WiLLa T ReiD 


\title{
RICCATI MATRIX DIFFERENTIAL EQUATIONS AND NON-OSCILLATION CRITERIA FOR ASSOCIATED LINEAR DIFFERENTIAL SYSTEMS
}

\author{
WILLIAM T. REID
}

1. Introduction. For real scalar linear homogeneous differential equations of the second order which are non-oscillatory on some interval $(a, \infty)$ the concept of a "principal solution at $\infty$ " was introduced by Leighton and Morse [4; 5]. Several years later Hartman and Wintner [2] studied the same concept, and subsequently Hartman [3] extended the notion of a principal solution to a self-adjoint matrix differential equation of the second order, characterizing such solutions by a distinguishing property in the class of solutions non-singular on some neighborhood of $\infty$ and which are "prepared" in his terminology. For a self-adjoint matrix differential system of more general type than considered by Hartman, Reid [9] presented a generalized definition of princiral solution that distinguishes such solutions in the class of all solutions that are non-singular on some neighborhood of $\infty$; the determination of principal solutions in [9] is based on variational methods which are applicable directly to differential systems with complex coefficients that are of the form of the accessory differential equations for a calculus of variations problem of Bolza type, (see, for example, Bliss [1, § 81]).

Recently S. Sandor [11] has considered properties of solutions of Riccati matrix differential equations, including a generalization of the classical anharmonic ratio property that in character is quite different from the generalization studied by Whyburn [12] and Reid [7]. Moreover, for a real self-adjoint matrix system equivalent to the equation considered by Hartman [3], Sandor has shown the equivalence of the existence of a principal solution at $\infty$ in the sense of Hartman and the existence of a "right-hand frontier solution" of the associated Riccati matrix differential equation. Evidently Sandor was unaware of the paper [9] of Reid, for there are many intimate relationships between the results of the two papers, although the method of attack is quite different.

The purrose of the present paper is to study in more detail the concept of a principal solution of a non-oscillatory linear matrix differential system, together with related problems for the associated Riccati matrix equation. In particular, certain aspects considered

Received August 21, 1962. This research was supported by the Air Force Office of Scientific Research, under Grant AF-AFOSR-62-78. 
previously in variational context only are here divorced from such limitations. $\S 2$ is devoted to basic relationships between linear matrix systems and Riccati equations, together with a representation theorem, (Lemma 2.1), which is derived under more general conditions than those employed by Sandor [11] in corresponding results, and which permits simplification in the ensuing proof of the anharmonic ratio property, of Sandor [11] and Levin [6]. The results in $\S 3$ on the variation of solutions of a Riccati equation are prefatory to $\S 4$ on the concepts of a "principal solution" for a non-oscillatory linear system, and the corresponding "distinguished solution" of the associated Riccati equation; in this discussion these concepts are not limited to the instance of self-adjoint linear systems, as has been the case in the above cited papers. $\S 5$ is devoted to the case in which the involved linear system is self-adjoint, but of a more general character than those treated by Hartman [3], Reid [9], and Sandor [11]. Systems that are non-oscillatory on intervals of the form $(-\infty, a)$ or $(-\infty, \infty)$ are treated briefly in $\S 6$, and $\S 7$ is devoted to certain specific results for systems with constant coefficients.

For simplicity of treatment, throughout the discussion of nonoscillatory systems in $\S 4-7$ it is assumed that the involved linear system is identically normal. For systems that are not identically normal, however, certain modifications of the basic theorems of $\S 4$, 5 hold, and the author plans to further this study in a subsequent paper.

Matrix notation is used throughout; in particular, matrices of one column are termed vectors, and for a vector $y=\left(y_{\alpha}\right),(\alpha=1, \cdots, n)$, the norm $|y|$ is given by $\left(\left|y_{1}\right|^{2}+\cdots+\left|y_{n}\right|^{2}\right)^{1 / 2}$. The symbol $E$ is used for the $n \times n$ identity matrix, while 0 is used indiscriminately for the zero matrix of any dimensions; the conjugate transpose of a matrix $M$ is denoted by $M^{*}$. If $M$ is an $n \times n$ matrix the symbol $|M|$ is used for the supremum of $|M y|$ on the unit sphere $|y|=1$. The notation $M \geqq N,\{M>N\}$, is used to signify that $M$ and $N$ are hermitian matrices of the same dimensions and $M-N$ is a nonnegative, \{positive\}, definite hermitian matrix. If $M \geqq 0$ then $M^{1 / 2}$ signifies the unique nonnegative definite square root of $M$; if $M>0$ then $M^{-1 / 2}$ denotes the reciprocal of $M^{1 / 2}$. For an arbitrary square matrix $M$ we set $M_{\Re}=\frac{1}{2}\left(M+M^{*}\right)$ and $M_{\widetilde{⿰}}=\frac{1}{2} i\left(M^{*}-M\right)$, so that $M_{\Re}$ and $M_{\Im}$ are the hermitian matrices with the definitive property $M=$ $M_{\Re}+i M_{\Im}$. If the elements of a matrix $M(x)$ are a.c. (absolutely continuous) on an interval $[c, d]$, then $M^{\prime}(x)$ signifies the matrix of derivatives at values for which these derivatives exist and the zero matrix elsewhere; correspondingly, if the elements of $M(x)$ are (Lebesgue) integrable on $[c, d]$ then $\int_{c}^{a} M(x) d x$ denotes the matrix of integrals 
of respective elements of $M(x)$. If matrices $M(x)$ and $N(x)$ are equal a.e. (almost everywhere) on their domain of definition we write simply $M(x)=N(x)$. Finally, for brevity a matrix $M(x)$ is termed continuous, etc., when each element of the matrix possesses the specified property.

2. Related linear systems and Riccati equations. The linear vector differential systems to be considered are of the form

$$
u^{\prime}=A(x) u+B(x) v, \quad v^{\prime}=C(x) u-D(x) v,
$$

where $u(x)$ and $v(x)$ are $n$-dimensional vector functions, and $A(x), B(x)$, $C(x)$ and $D(x)$ are $n \times n$ matrices with complex elements which are (Lebesgue) integrable on arbitrary compact subintervals of a given interval $X$ on the real line. A major portion of our discussion involves the corresponding matrix differential equations

$$
U^{\prime}=A(x) U+B(x) V, \quad V^{\prime}=C(x) U-D(x) V,
$$

where in general $U(x)$ and $V(x)$ are matrices of $n$ rows and $r,(r \geqq$ $1)$, columns. By a solution $(u ; v)$ of $(2.1)$, or a solution $(U ; V)$ of (2.2), will be meant vector or matrix functions which are a.c. on arbitrary compact subintervals of $X$, and such that (2.1) or (2.2) hold a.e. on $X$. For brevity, we introduce the notations

$$
\begin{aligned}
& L_{1}[U, V]=U^{\prime}-A(x) U-B(x) V, \\
& L_{2}[U, V]=V^{\prime}-C(x) U+D(x) V,
\end{aligned}
$$

for general $n$-rowed matrices $U, V$ so that (2.2) becomes $L_{\alpha}[U, V]=$ $0, \alpha=1,2$.

If $U(x), V(x)$ are $n \times n$ matrix functions a.c. on compact subintervals of $X$, and $U(x)$ is non-singular on $X$, then the corresponding Riccati matrix differential operator

$$
K[W] \equiv W^{\prime}+W A(x)+D(x) W+W B(x) W-C(x)
$$

satisfies the identity

$$
U^{*}(x) K\left[V U^{-1}\right] U(x) \equiv U^{*}(x)\left(L_{2}[U, V]-V(x) U^{-1}(x) L_{1}[U, V]\right) .
$$

Consequently, if $(U(x) ; V(x))$ is a solution of (2.2) on $X$ with $U(x)$ nonsingular on this interval, then $W(x)=V(x) U^{-1}(x)$ is a solution of the Riccati matrix differential equation

$$
K[W]=0
$$

on this interval; that is, $W(x)$ is an $n \times n$ matrix which is a.c. on compact subintervals of $X$ and (2.6) holds a.e. on $X$. Conversely, if $W(x)$ is a solution of (2.6) on $X$, and for $s \in X$ the matrix $U(x)$ is 
determined as the solution of

$$
U^{\prime}=[A(x)+B(x) W(x)] U, \quad U(s)=M, \quad M \text { nonsingular },
$$

then $(U ; V)=(U(x) ; W(x) U(x))$ is the solution of (2.2) satisfying $U(s)=M, V(s)=W(s) M$, and $W(x)=V(x) U^{-1}(x)$ on $X$.

If $W(x)$ and $W_{0}(x)$ are $n \times n$ matrices a.c. on compact subintervals. of $X$, then $\Psi(x)=W(x)-W_{0}(x)$ satisfies the identity

$$
K[W]-K\left[W_{0}\right]=\Psi^{\prime}+\Psi\left(A+B W_{0}\right)+\left(D+W_{0} B\right) \Psi+\Psi B \Psi .
$$

Lemma 2.1. If $W_{0}(x)$ is a solution of (2.6) on $X$, and for $s \in X$ the matrices $G(x)=G\left(x, s \mid W_{0}\right), H(x)=H\left(x, s \mid W_{0}\right)$ are solutions of the linear differential systems

$$
\begin{aligned}
& G^{\prime}+\left(D+W_{0} B\right) G=0, \quad G(s)=E, \\
& H^{\prime}+H\left(A+B W_{0}\right)=0, \quad H(s)=E,
\end{aligned}
$$

and

$$
\Theta\left(x, s \mid W_{0}\right)=\int_{s}^{x} H(t) B(t) G(t) d t,
$$

then $W(x)$ is a solution of (2.6) on $X$ if and only if the constant matrix $\Gamma=W(s)-W_{0}(s)$ is such that $E+\epsilon\left(x, s \mid W_{0}\right) \Gamma$ is nonsingular on $X$, and

$$
W(x)=W_{0}(x)+G\left(x, s \mid W_{0}\right) \Gamma\left[E+\Theta\left(x, s \mid W_{0}\right) \Gamma\right]^{-1} H\left(x, s \mid W_{0}\right) .
$$

If $K\left[W_{0}\right]=0$ on $X$, and for an arbitrary $W(x)$ we set $\Psi(x)=$ $W(x)-W_{0}(x)$, in view of (2.7), (2.8), (2.9) it follows that $W$ satisfies (2.6) on $X$ if and only if the matrix $F(x)$ defined by $\Psi(x)=G(x) F(x) H(x)$ is a solution on $X$ of the special Riccati matrix differential equation.

$$
F^{\prime}+F[H(x) B(x) G(x)] F=0, \quad F(s)=\Gamma \equiv W(s)-W_{0}(s) .
$$

If $F(x)$ is a solution of (2.12) on $X$, and $\epsilon\left(x, s \mid W_{0}\right)$ is defined by (2.10), then $F_{1}(x)=F(x)\left[E+\epsilon\left(x, s \mid W_{0}\right) \Gamma\right]-\Gamma$ satisfies the linear homogeneous system

$$
F_{1}^{\prime}=-F(x) H(x) B(x) G(x) F_{1}, \quad F_{1}(s)=0,
$$

and consequently $F_{1}(x) \equiv 0$ on $X$. Moreover, if $r \in X$ and $\eta$ is a vector such that $\left[E+\epsilon\left(r, s \mid W_{0}\right) \Gamma\right] \eta=0$, then $0=F_{1}(r) \eta=-\Gamma \eta$, and hence $\eta=0$. Consequently $E+\epsilon\left(x, s \mid W_{0}\right) \Gamma$ is nonsingular throughout $X_{\text {p }}$ and

$$
F(x)=\Gamma\left[E+E\left(x, s \mid W_{0}\right) \Gamma\right]^{-1}
$$

on this interval. Conversely, if $\Gamma$ is a constant matrix such that 
$E+\Theta\left(x, s \mid W_{0}\right) \Gamma$ is nonsingular throughout $X$, then $F(x)$ defined by (2.14) is the solution of (2.12) on $X$, and $W(x)$ given by (2.11) satisfies (2.6).

Since for arbitrary $n \times n$ matrices $\Theta, \Gamma$ the identity $(E+\Gamma \Theta) \Gamma=$ $\Gamma(E+\Theta \Gamma)$ implies that $E+\Gamma \Theta$ is non-singular if and only if $E+\Theta \Gamma$ is non-singular, and $\Gamma(E+\Theta \Gamma)^{-1}=(E+\Gamma \Theta)^{-1} \Gamma$, the non-singularity of $E+\epsilon\left(x, s \mid W_{0}\right) \Gamma$ on $X$ is equivalent to the non-singularity of $E+$ $\Gamma \Theta\left(x, s \mid W_{0}\right)$ on this interval, and an alternate form of (2.11) is

$$
W(x)=W_{0}(x)+G\left(x, s \mid W_{0}\right)\left[E+\Gamma \Theta\left(x, s \mid W_{0}\right)\right]^{-1} \Gamma H\left(x, s \mid W_{0}\right) .
$$

In particular, if $W_{0}(x)$ and $W(x)$ are solutions of (2.6) on $X$, and $\Gamma=W(s)-W_{0}(s)$ is non-singular, then (2.11) and (2.11') each reduces to

$\left(2.11^{\prime \prime}\right) \quad W(x)=W_{0}(x)+G\left(x, s \mid W_{0}\right)\left[\Gamma^{-1}+\Theta\left(x, s \mid W_{0}\right)\right]^{-1} H\left(x, s \mid W_{0}\right)$.

For the special case of $\Gamma=W(s)-W_{0}(s)$ non-singular Sandor [11] obtained this latter formula, and in this instance he termed $W(x)$ representable with the aid of $W_{0}(x)$ by $\left(2.11^{\prime}\right)$. The above results presenting (2.11) and (2.11') show that this concept of representability may be given a form independent of the non-singularity of $W(s)-W_{0}(s)$. Moreover, it is to be noted that (2.11) implies that throughout $X$ the rank of $W(x)-W_{0}(x)$ is equal to that of $\Gamma$, thus presenting a new proof of the known result that the difference of two solutions of (2.6) is of constant rank throughout a common interval of definition, (see Reid [7; Theorem 2.1]). Finally, it is to be remarked that if $W_{0}(x)$ is a solution of (2.6) on an interval $X$, and $\left(U_{0}(x) ; V_{0}(x)\right)$ is a solution of (2.2) such that $U_{0}(x)$ is nonsingular and $W_{0}(x)=V_{0}(x) U_{0}^{-1}(x)$ on this interval, then the solution $H\left(x, s \mid W_{0}\right)$ of (2.9) is given by

$$
H\left(x, s \mid W_{0}\right)=U_{0}(s) U_{0}^{-1}(x) .
$$

LEMMA 2.2. If $W_{0}(x), W_{\alpha}(x),(\alpha=1, \cdots, k)$, are solutions of (2.6) on $X, s \in X$, and $\Gamma_{a}=W_{\alpha}(s)-W_{0}(s)$, then

$$
\begin{aligned}
W_{\alpha}(x)-W_{\beta}(x)= & G\left(x, s \mid W_{0}\right)\left[E+\Gamma_{\beta} \Theta\left(x, s \mid W_{0}\right)\right]^{-1} \\
& \cdot\left(\Gamma_{\alpha}-\Gamma_{\beta}\right)\left[E+\theta\left(x, s \mid W_{0}\right) \Gamma_{\alpha}\right]^{-1} H\left(x, s \mid W_{0}\right) .
\end{aligned}
$$

In view of Lemma 2.1, $G=G\left(x, s \mid W_{0}\right), H=H\left(x, s \mid W_{0}\right)$ and $\Theta=$ $\theta\left(x, s \mid W_{0}\right)$ are such that

$$
W_{\alpha}-W_{0}=G \Gamma_{\alpha}\left[E+\Theta \Gamma_{\alpha}\right]^{-1} H=G\left[E+\Gamma_{a} \Theta\right]^{-1} \Gamma_{\alpha} H,
$$

and (2.16) is an immediate consequence of the relation

$$
\begin{aligned}
\Gamma_{\alpha}-\Gamma_{\beta} & =\left[E+\Gamma_{\beta} \Theta\right] \Gamma_{\alpha}-\Gamma_{\beta}\left[E+\Theta \Gamma_{\alpha}\right], \\
& =\left[E+\Gamma_{\beta} \Theta\right]\left(\Gamma_{\alpha}\left[E+\Theta \Gamma_{\alpha}\right]^{-1}-\left[E+\Gamma_{\beta} \Theta\right]^{-1} \Gamma_{\beta}\right)\left[E+\Theta \Gamma_{\alpha}\right] .
\end{aligned}
$$


If $M_{1}, M_{2}, M_{3}, M_{4}$ are $n \times n$ matrices with $M_{3}-M_{2}$ and $M_{4}-M_{0}$ non-singular, we introduce the notations

$$
\begin{aligned}
& \left\{M_{1}, M_{2}, M_{3}\right\}=\left(M_{3}-M_{1}\right)\left(M_{3}-M_{2}\right)^{-1} \\
& \begin{aligned}
\left\{M_{1}, M_{2}, M_{3}, M_{4}\right\} & =\left\{M_{1}, M_{2}, M_{3}\right\}\left\{M_{2}, M_{1}, M_{4}\right\} \\
& =\left(M_{3}-M_{1}\right)\left(M_{3}-M_{2}\right)^{-1}\left(M_{4}-M_{2}\right)\left(M_{4}-M_{1}\right)^{-1}
\end{aligned}
\end{aligned}
$$

Clearly, $\left\{M_{1}, M_{2}, M_{3}, M_{4}\right\}$ is a direct matrix generalization of the scalar anharmonic ratio.

THEOREM 2.1. If $W_{0}(x), W_{\alpha}(x),(\alpha=1,2,3,4)$, are solutions of (2.6) on $X$ with $W_{3}(x)-W_{2}(x)$ and $W_{4}(x)-W_{1}(x)$ non-singular, and $s \in X$, then

$$
\begin{aligned}
& \left\{W_{1}(x), W_{2}(x), W_{3}(x), W_{4}(x)\right\} \\
& \quad=\Phi\left(x, s \mid W_{0}, W_{1}\right)\left\{W_{1}(s), W_{2}(s), W_{3}(s), W_{4}(s)\right\} \Phi^{-1}\left(x, s \mid W_{0}, W_{1}\right),
\end{aligned}
$$

where $\phi\left(x, s \mid W_{0}, W_{1}\right)=G\left(x, s \mid W_{0}\right)\left[E+\left.\Gamma_{1} \theta\left(x, s \mid W_{0}\right)\right|^{-1}\right.$, and $\Gamma_{1}=$ $W_{1}(s)-W_{0}(s)$.

If $\Gamma_{\alpha}=W_{\alpha}(s)-W_{0}(s),(\alpha=1,2,3,4)$, then from (2.16) it follows directly that $G=G\left(x, s \mid W_{0}\right)$ satisfies

$$
\begin{aligned}
& \left\{W_{1}(x), W_{2}(x), W_{3}(x)\right\}=G\left[E+\Gamma_{1}^{r} \Theta\right]^{-1}\left\{\Gamma_{1}, \Gamma_{2}, \Gamma_{3}\right\}\left[E+\Gamma_{2} \Theta\right] G^{-1}, \\
& \left\{W_{2}(x), W_{1}(x), W_{4}(x)\right\}=G\left[E+\Gamma_{2} \Theta\right]^{-1}\left\{\Gamma_{2}, \Gamma_{1}, \Gamma_{4}\right\}\left[E+\Gamma_{1} \Theta\right] G^{-1},
\end{aligned}
$$

and (2.18) is an immediate consequence of these relations and

$$
\left\{I_{1}^{r}, \Gamma_{2}, \Gamma_{3} \Gamma_{4}{ }_{4}\right\}=\left\{W_{1}(\mathrm{~s}), W_{2}(s), W_{3}(s), W_{4}(s)\right\} .
$$

The fact that the "anharmonic ratio" of four solutions of (2.6) is similar to a constant matrix has been established by Sander [11] and Levin [6]; it is to be noted that Levin's hypotheses are needlessly strong as he supposes that $W_{\alpha}(x)-W_{\beta}(x),(\alpha, \beta=1,2,3,4 ; \alpha \neq \beta)$, is non-singular. In view of the generality of the result of our Lemma 2.2, however, the proof of the above Theorem 2.1 is more direct than that given by Sandor for his Theorem 1, which involved the determination of a particular solution $W_{0}(x)$ such that each of the constant matrices $I_{\alpha}^{\prime},(\alpha=1,2,3,4)$, is non-singular. Indeed, in the proof of Theorem 2.1 one might choose $W_{0}(x)=W_{1}(x)$, in which case $\Gamma_{1}=0$ and (2.18) reduces to

$$
\begin{aligned}
& \left\{W_{1}(x), W_{2}(x), W_{3}(x), W_{4}(x)\right\} \\
& \quad=G\left(x, s \mid W_{1}\right)\left\{W_{1}(s), W_{2}(s), W_{3}(s), W_{4}(s)\right\} G^{-1}\left(x, s \mid W_{1}\right) .
\end{aligned}
$$

It is to be remarked that the above type of anharmonic ratio property of four solutions of (2.6) is quite different from the generalization of the anharmonic ratio considered by Whyburn [12] and Reid [7]. 
With the aid of (2.18) and (2.19) one may deduce that if $W_{0}(x)$ and $W_{1}(x)$ are solutions of $(2.6)$ on an interval $X$ then

$$
\begin{aligned}
G\left(x, s \mid W_{1}\right) & =G\left(x, s \mid W_{0}\right)\left[E+\Gamma_{1} \Theta\left(x, s \mid W_{0}\right)\right]^{-1}, \\
\Gamma_{1} & =W_{1}(s)-W_{0}(s) .
\end{aligned}
$$

where

Relation (2.20) is but one of the variational relations for solutions of (2.6) which will be established in the next section, however, so it will not be considered further here.

Of special significance is the class of systems for which the coefficient matrices satisfy the conditions

$$
B(x) \equiv B^{*}(x), \quad C(x) \equiv C^{*}(x), \quad D(x) \equiv A^{*}(x),
$$

since particular systems of this type occur as accessory systems for simple integral variational problems, (see, for example, Bliss [1, § 81], Reid [7]). In this instance, if $(U(x) ; V(x))$ is a solution of (2.2) on $X$ then there exists a constant matrix $K$ such that $U^{*}(x) V(x)-$ $V^{*}(x) U(x) \equiv K$; in particular, if $U(x)$ is non-singular on $X$ then $W(x)=V(x) U^{-1}(x)$ is a solution of (2.6) on $X$ such that

$$
W(x)-W^{*}(x)=U^{*-1}(x) K U^{-1}(x),
$$

and $W(x)$ is hermitian if and only if $K=0$. Moreover, if $s \in X$ then the solution $H=H(x, s \mid W)$ of the corresponding equation (2.9) satisfies

$$
H^{* \prime}+\left(D+\left[W-U^{*-1} K U^{-1}\right] B\right) H^{*}=0, H^{*}=E \text { for } x=s,
$$

and for the solution $G=G(x, s \mid W)$ of the corresponding equation (2.8) the relation (2.15) and the method of variation of parameters yields

$$
G(x, s \mid W)=H^{*}(x, s \mid W) U^{*-1}(s) T^{*-1}(x, s ; U) U^{*}(s),
$$

where $T=T(x, s \mid U)$ is the solution of the differential system

$$
T^{\prime}=-U^{-1}(x) B(x) U^{*-1}(x) K T, \quad T(s)=E .
$$

Consequently the function $\Theta(x, s \mid W)$ given by (2.10) has the form

$$
\Theta(x, s \mid W)=U(s) S^{*}(x, s ; U) U^{*}(s),
$$

where

$$
S(x, s ; U)=\int_{s}^{x} T^{-1}(t, s ; \quad U) U^{-1}(t) B(t) U^{*-1}(t) d t
$$

is the function introduced by Reid [9, equation (3.6)] for the general characterization of principal solutions of non-oscillatory self-adjoint differential systems. 
Following the terminology used by Reid $[8 ; 9]$, if the coefficient matrices satisfy (2.21) then two solutions $\left(u_{1}(x) ; v_{1}(x)\right)$ and $\left(u_{2}(x) ; v_{2}(x)\right)$ of (2.1) for which the constant value of $u_{1}^{*}(x) v_{2}(x)-v_{1}^{*}(x) u_{2}(x)$ is zero are said to be (mutually) conjoined solutions. As in Lemma 2.3 of Reid [8], one may prove for such systems (2.1) that the maximum dimension of a conjoined family of solutions is $n$, and that a given conjoined family of solutions of dimension less than $n$ is contained in a conjoined family of dimension $n$.

3. Variation of solutions. Let $\boldsymbol{A}(x)$ denote the $2 n \times 2 n$ direct sum matrix

$$
\boldsymbol{A}(x)=\left\|\begin{array}{cc}
A(x) & 0 \\
0 & 0
\end{array}\right\|,
$$

where 0 is the $n \times n$ zero matrix, with similar definitions for $\boldsymbol{B}(x)$, $C(x), D(x)$ in terms of the corresponding $B(x), C(x), D(x)$. It may be verified directly that a $2 n \times 2 n$ matrix $W(x)$ is a solution of the Riccati matrix differential equation

$$
W^{\prime}+\boldsymbol{W A}(x)+D(x) W+W B(x) W-C(x)=0
$$

on an interval $X$ if and only if

$$
\boldsymbol{W}(x)=\left\|\begin{array}{lr}
W(x) & G(x) \\
H(x) & -\Theta(x)
\end{array}\right\|,
$$

where $W(x), G(x), H(x)$ and $\Theta(x)$ are $n \times n$ matrices which satisfy on this interval the Riccati system

$$
\begin{aligned}
& W^{\prime}+W A(x)+D(x) W+W B(x) W-C(x)=0, \\
& G^{\prime}+[D(x)+W B(x)] G=0, \\
& H^{\prime}+H[A(x)+D(x) W]=0, \\
& \Theta^{\prime}-H B(x) G=0 .
\end{aligned}
$$

This relation between a Riccati system (3.3) and the associated single Riccati equation (3.1) has been exploited previously by the author in the study of a different type of problem, (see Reid $[10, \S 4]$ ).

In particular, if $W_{0}(x)$ is a solution of (2.6) on $X$, and $G\left(x, s \mid W_{0}\right)$, $H\left(x, s \mid W_{0}\right)$ and $\epsilon\left(x, s \mid W_{0}\right)$ are defined by (2.8), (2.9) and (2.10), then the solution $\boldsymbol{W}=W_{0}(x)$ of (3.1) satisfying the initial condition

$$
W_{0}(s)=\left\|\begin{array}{cc}
W_{0}(s) & E \\
E & 0
\end{array}\right\|
$$

is given by

$$
\boldsymbol{W}_{0}(x)=\left\|\begin{array}{cc}
W_{0}(x) & G\left(x, s \mid W_{0}\right) \\
H\left(x, s \mid W_{0}\right) & -G\left(x, s \mid W_{0}\right)
\end{array}\right\| .
$$


Moreover, for this solution $W_{0}(x)$ of (3.1) the matrix functions $\boldsymbol{G}\left(x, s \mid \boldsymbol{W}_{0}\right), \boldsymbol{H}\left(x, s \mid \boldsymbol{W}_{0}\right), \Theta\left(x, s \mid \boldsymbol{W}_{0}\right)$ determined by the corresponding equations (2.8), (2.9), (2.10) are computed readily to be

$$
\begin{aligned}
& \boldsymbol{G}\left(x, s \mid \boldsymbol{W}_{0}\right)=\left\|\begin{array}{cc}
G\left(x, s \mid W_{0}\right) & 0 \\
-\Theta\left(x, s \mid W_{0}\right) & E
\end{array}\right\|, \\
& \boldsymbol{H}\left(x, s \mid \boldsymbol{W}_{0}\right)=\left\|\begin{array}{cc}
H\left(x, s \mid W_{0}\right) & -\Theta\left(x, s \mid W_{0}\right) \\
0 & E
\end{array}\right\|, \\
& \Theta\left(x, s \mid \boldsymbol{W}_{0}\right)=\left\|\begin{array}{cc}
\Theta\left(x, s \mid W_{0}\right) & 0 \\
0 & 0
\end{array}\right\| .
\end{aligned}
$$

If $W_{0}(x)$ is the solution (3.5) of (3.1) on $X$, and $W(x)$ is a second solution of this equation on $X$ satisfying the initial condition

$$
\boldsymbol{W}(s)=\left\|\begin{array}{cc}
W(s) & E \\
E & 0
\end{array}\right\|,
$$

then the associated equation (2.11) in $W(x)$ and $W_{0}(x)$, with

$$
\Gamma=\left\|\begin{array}{ll}
\Gamma & 0 \\
0 & 0
\end{array}\right\|, \quad \Gamma=W(s)-W_{0}(s),
$$

yields (2.11) in $W(x), W_{0}(x)$ and also the following additional equations of variation:

$$
\begin{aligned}
G(x, s \mid W) & =G\left(x, s \mid W_{0}\right)\left[E+\Gamma \Theta\left(x, s \mid W_{0}\right)\right]^{-1}, \\
H(x, s \mid W) & =\left[E+\Theta\left(x, s \mid W_{0}\right) \Gamma\right]^{-1} H\left(x, s \mid W_{0}\right), \\
\Theta(x, s \mid W) & =\left[E+\Theta\left(x, s \mid W_{0}\right) \Gamma\right]^{-1} \Theta\left(x, s \mid W_{0}\right), \\
& =\Theta\left(x, s \mid W_{0}\right)\left[E+\Gamma \Theta\left(x, s \mid W_{0}\right)\right]^{-1} .
\end{aligned}
$$

In particular, if $\Theta\left(x, s \mid W_{0}\right)$ is non-singular on a subinterval $X_{0}$ of $X$ then $\Theta(x, s \mid W)$ is non-singular on this subinterval also, and

$$
\Theta^{-1}(x, s \mid W)=\Theta^{-1}\left(x, s \mid W_{0}\right)+\Gamma .
$$

4. Principal solutions for non-oscillatory systems (2.1.) Two distinct points $s$ and $t$ on $X$ are said to be (mutually) conjugate, (with respect to (2.1)) if there exists a solution $(u(x) ; v(x))$ of this system with $u(x) \neq 0$ on the subinterval with endpoints $s$ and $t$, while $u(s)=$ $0=u(t)$. The system (2.1) is termed non-oscillatory on a given subinterval $X_{0}$ provided no two distinct points of this subinterval are conjugate; moreover, (2.1) will be called non-oscillatory for large \{small $\} x$ if there exists a subinterval $[a, \infty)\left\{\left(-\infty, a_{1}\right]\right\}$ of $X$ on which this system is non-oscillatory.

A system (2.1) is termed identically normal on $X$, or normal on every subinterval of $X$, if whenever $(u ; v)=(0 ; v(x))$ is a solution of 
this system on a non-degenerate subinterval of $X$ then also $v(x) \equiv 0$ on this subinterval. If (2.1) is identically normal on $X, s \in X$, and $(U(x) ; V(x))$ is a solution of (2.2) with $U(s)=0$ and $V(s)$ non-singular, then the points $t$ conjugate to $s$ are those values for which $U(t)$ is singular; in particular, if such a system is non-oscillatory on an interval $X$, and $s \in X$, then $U(x)$ is non-singular on each of the subintervals $X_{s}^{+}=\{x \mid x \in X, x>s\}$ and $X_{s}^{-}=\{x \mid x \in X, x<s\}$.

A basic result for non-oscillatory systems is the following theorem. It is to be emphasized that in contrast to the special case considered previously by the author in [9], the result of this theorem is not limited to self-adjoint systems of the form of accessory equations for problems of the calculus of variations, and the proof is independent of variational principles.

THEOREM 4.1. If (2.1) is identically normal and non-oscillatory on an interval $X$, and $W_{0}(x)$ is a solution of (2.6) on this interval, then for $s \in X$ the matrix $\Theta\left(x, s \mid W_{0}\right)$ is non-singular on each of the subintervals $X_{s}^{+}$and $X_{s}^{-}$; moreover,

$$
\Theta^{-1}\left(t, s \mid W_{0}\right)=W_{0}(s)-W_{t}(s), \quad t \in X_{s}^{+} \text {or } t \in X_{s}^{-},
$$

where $W_{t}(x)=V_{t}(x) U_{t}^{-1}(x)$ and $\left(U_{t}(x) ; V_{t}(x)\right)$ is the solution of (2.2) determined by the initial conditions

$$
U_{t}(t)=0, \quad V_{t}(t)=E .
$$

Suppose that $t \in X_{s}^{+}$, and $\left(U_{t}(x) ; V_{t}(x)\right)$ is the solution of (2.2) satisfying (4.2). In view of the condition that (2.1) is identically normal and non-oscillatory on $X$, the matrix $U_{t}(x)$ is non-singular on $X_{t}^{-}$and $X_{t}^{+}$. In particular, on $X_{t}^{-}$each of the matrices $W_{0}(x)$ and $W_{t}(x)=V_{t}(x) U_{t}^{-1}(x)$ is a solution of (2.6), $H\left(x, s \mid W_{t}\right)=U_{t}(s) U_{t}^{-1}(x)$, and from (3.9) we have

$$
U_{t}(s) U_{t}^{-1}(x)=\left[E+\Theta\left(x, s \mid W_{0}\right)\left\{W_{t}(s)-W_{0}(s)\right\}\right]^{-1} H\left(x, s \mid W_{0}\right), x \in X_{t}^{-} .
$$

Consequently,

$$
\left[E+\Theta\left(x, s \mid W_{0}\right)\left\{W_{t}(s)-W_{0}(s)\right\}\right] U_{t}(s)=H\left(x, s \mid W_{0}\right) U_{t}(x), x \in X_{t}^{-},
$$

and by continuity (4.3) also holds for $x=t$. As $s \in X_{t}^{-}$and $U_{t}(s)$ is non-singular, while $U_{t}(t)=0$, it follows that $\Theta\left(t, s \mid W_{0}\right)$ is non-singular with inverse $W_{0}(s)-W_{t}(s)$, so that $\Theta\left(x, s \mid W_{0}\right)$ is non-singular for $x \in X_{s}^{+}$. A similar argument shows that $\Theta\left(t, s \mid W_{0}\right)$ is non-singular and (4.1) holds for $t \in X_{s}^{-}$.

It is to be emphasized that the non-oscillation of (2.1) on $X$ is not a consequence of the existence of a solution $W_{0}(x)$ of (2.6), or the equivalent condition that there is a solution $\left(U_{0}(x) ; V_{0}(x)\right)$ of (2.2) 
with $U_{0}(x)$ non-singular throughout $X$. Indeed, for any self-adjoint system (2.1) with coefficient matrices satisfying (2.21) the existence of a solution $\left(U_{0}(x) ; V_{0}(x)\right)$ with $U_{0}(x)$ non-singular throughout $X$ is illustrated by any solution $\left(U_{0}(x) ; V_{0}(x)\right)$ satisfying at an initial point $s$ the condition $U_{0}^{*}(s) V_{0}(s)-V_{0}^{*}(s) U_{0}(s)=i K_{0}$, where $K_{0}$ is a definite hermitian matrix. On the other hand, for the general system (2.1) that is identically normal and non-oscillatory on $X$ the author has not settled the question as to the existence of a solution $W_{0}(x)$ of $(2.6)$ throughout $X$.

It $W_{0}(x)$ is a solution of (2.6) on $X$, the semi-group properties

$$
\begin{aligned}
& G\left(x, s \mid W_{0}\right)=G\left(x, t \mid W_{0}\right) G\left(t, s \mid W_{0}\right), \\
& H\left(x, s \mid W_{0}\right)=H\left(t, s \mid W_{0}\right) H\left(x, t \mid W_{0}\right), \quad s, t, x \in X
\end{aligned}
$$

of the solutions of (2.8), (2.9) imply for $\Theta\left(x, s \mid W_{0}\right)$ of (2.10) the relation

$$
\Theta\left(x, s \mid W_{0}\right)=\Theta\left(t, s \mid W_{0}\right)+H\left(t, s \mid W_{0}\right) \Theta\left(x, t \mid W_{0}\right) G\left(t, s \mid W_{0}\right) .
$$

Since for an identically normal system that is non-oscillatory on $X$ we have $\Theta\left(x, s \mid W_{0}\right)$ non-singular for $x \neq s$, from (4.5) it follows that for $x \in X$ and distinct from both $t$ and $s$ the matrix

$$
\chi\left(x, t, s \mid W_{0}\right)=E+H^{-1}\left(t, s \mid W_{0}\right) \Theta\left(t, s \mid W_{0}\right) G^{-1}\left(t, s \mid W_{0}\right) \Theta^{-1}\left(x, t \mid W_{0}\right)
$$
is non-singular, and

$$
\text { (4.7) } \Theta^{-1}\left(x, s \mid W_{0}\right)=G^{-1}\left(t, s \mid W_{0}\right) \Theta^{-1}\left(x, t \mid W_{0}\right) \chi^{-1}\left(x, t, s \mid W_{0}\right) H^{-1}\left(t, s \mid W_{0}\right) \text {. }
$$

From (4.6), (4.7) it follows that if $\Theta^{-1}\left(x, t \mid W_{0}\right) \rightarrow 0$ as $x \rightarrow \infty$, then also $\Theta^{-1}\left(x, s \mid W_{0}\right) \rightarrow 0$ as $x \rightarrow \infty$; moreover, for $X_{0}$ an arbitrary compact subinterval of $X$ it follows from (4.7) that the convergence of $\Theta^{-1}\left(x, s \mid W_{0}\right)$ to 0 as $x \rightarrow \infty$ is uniform for $s$ on $X_{0}$.

For an identically normal system that is non-oscillatory for large $x$ a solution $\left(U_{\infty}(x) ; V_{\infty}(x)\right)$ will be termed a principal solution at $\infty$ for (2.2) if $U_{\infty}(x)$ is non-singular on some subinterval $(a, \infty)$ and for $W_{\infty}(x)=V_{\infty}(x) U_{\infty}^{-1}(x)$ we have $\Theta^{-1}\left(x, s \mid W_{\infty}\right) \rightarrow 0$ as $x \rightarrow \infty$ for at least one, (and consequently all), $s$ on $(a, \infty)$; the corresponding solution $W_{\infty}(x)$ of (2.6) will be called a distinguished solution at $\infty$ of this Riccati equation.

THEOREM 4.2. If for an identically normal system (2.1) that is non-oscillatory for large $x$ there exists a principal solution $\left(U_{\infty}(x)\right.$; $\left.V_{\infty}(x)\right)$ with $U_{\infty}(x)$ non-singular on $[a, \infty)$, then: $(a) U_{\infty}(x), V_{\infty}(x)$ and $W_{\infty}(x)=V_{\infty}(x) U_{\infty}^{-1}(x)$ are such that as $t \rightarrow \infty$,

$$
\begin{aligned}
& W_{t}(s) \rightarrow W_{\infty}(s), \quad U_{t}(s) U_{t}^{-1}(a) U_{\infty}(a) \rightarrow U_{\infty}(s), \\
& V_{t}(s) U_{t}^{-1}(a) U_{\infty}(a) \rightarrow V_{t}(s)
\end{aligned}
$$


uniformly for $s$ on an arbitrary compact subinterval of $[a, \infty)$, where, as in Theorem 4.1, $\left(U_{t}(x) ; V_{t}(x)\right)$ is the solution of (2.2) satisfying (4.2) and $W_{t}(x)=V_{t}(x) U_{t}^{-1}(x)$; (b) the associated distinguished solution of (2.6) at $\infty$ is determined uniquely and the most general principal solution of $(2.2)$ at $\infty$ is $\left(U_{\infty}(x) M ; V_{\infty}(x) M\right)$, where $M$ is a non-singular constant matrix.

Equation (4.1) and the remark following (4.7) imply that for a principal solution $\left(U_{\infty}(x) ; V_{\infty}(x)\right)$ of $(2.2)$ the associated distinguished solution $W_{\infty}(x)=V_{\infty}(x) U_{\infty}^{-1}(x)$ of $(2.6)$ is such that $W_{t}(s) \rightarrow W_{\infty}(s)$ uniformly in $s$ on an arbitrary compact subinterval of $[a, \infty)$. The second limit relation of (4.8), and the uniformity of this limit on arbitrary compact subsets, follow from the preceding limit relation and the fact that $U_{t}^{0}(x)=U_{t}(x) U_{t}^{-1}(a) U_{\infty}(a)$ and $U_{\infty}(x)$ are solutions of the differential systems

$$
\begin{aligned}
U_{t}^{0 \prime} & =\left[A(x)+B(x) W_{t}(x)\right] U_{t}^{0}, \\
U_{\infty}^{\prime} & =\left[A(x)+B(x) W_{\infty}(x)\right] U_{\infty},
\end{aligned}
$$

and $U_{t}^{0}(a)=U_{\infty}(\alpha)$. In turn, the last limit relation of (4.8) and the stated property of uniformity are immediate consequences of the first two limits of (4.8) and the respective uniformity properties. Finally, the uniqueness of a distinguished solution of (2.6) at $\infty$, and the most general form of a principal solution for (2.2), are direct consequences of relations (4.8).

As will be shown in the next section, for a class of identically normal self-adjoint systems more inclusive than those previously studied by Hartman [3], Reid [9] and Sandor [11] the condition of non-oscillation for large $x$ implies the existence of a principal solution of (2.2) at $\infty$. Such is not true for systems in general, however, as is illustrated by the simple scalar system

$$
u^{\prime}=v, \quad v^{\prime}=\left[h^{\prime \prime}(x) / h^{\prime}(x)\right] v, \quad 0 \leqq x<\infty,
$$

where $h(x)$ is a function of class $C^{\prime \prime}$ on $[0, \infty)$ with

$$
h^{\prime}(x) \neq 0, \quad h\left(x_{1}\right) \neq h\left(x_{2}\right) \text { for } x_{1} \neq x_{2}, \quad 0 \leqq x<\infty .
$$

The general solution of (4.9) is $u=c_{1}+c_{2} h(x), v=c_{2} h^{\prime}(x)$, and the associated Riccati differential equation

$$
w^{\prime}-\left[h^{\prime \prime}(x) / h^{\prime}(x)\right] w+w^{2}=0
$$

has as solution $w(x)=\left[c_{2} h^{\prime}(x)\right] /\left[c_{1}+c_{2} h(x)\right]$ throughout any interval where $c_{1}+c_{2} h(x) \neq 0$. In particular, if $w=w_{0}(x)$ is a solution of (4.11) on an interval $[a, \infty)$, then either $w_{0}(x) \equiv 0$ or $w_{0}(x)=$ $h^{\prime}(x) /[h(x)-c]$, where $c$ is a constant such that $h(x) \neq c$ on this 
interval. If $w_{0}(x) \equiv 0$ then $w_{0}(x)=v_{0}(x) u_{0}^{-1}(x)$, where $u_{0}(x) \equiv k \neq 0$, $v_{0}(x) \equiv 0$ is a corresponding solution of (4.9), and $G\left(x, s \mid w_{0}\right)=h^{\prime}(x) / h^{\prime}(s)$, $H\left(x, s \mid w_{0}\right) \equiv 1$, and $\Theta^{-1}\left(x, s \mid w_{0}\right)=h^{\prime}(s) /[h(x)-h(s)]$, so that $\Theta^{-1}\left(x, s \mid w_{0}\right)$ $\rightarrow 0$ as $x \rightarrow \infty$ only if $|h(x)| \rightarrow \infty$ as $x \rightarrow \infty$. In case $w_{0}(x)=$ $h^{\prime}(x) /[h(x)-c]$, then $u_{0}(x)=k[h(x)-c]$ and $v_{0}(x)=k h^{\prime}(x)$ with $k \neq 0$, $G\left(x, s \mid w_{0}\right)=\left(h^{\prime}(x)[h(s)-c]\right) /\left(h^{\prime}(s)[h(x)-c]\right), \quad H\left(x, s \mid w_{0}\right)=[h(s)-c] /$ $[h(x)-c]$, and $\Theta^{-1}\left(x, s \mid w_{0}\right)=\left(h^{\prime}(s)[h(x)-c]\right) /([h(s)-c][h(x)-h(s)])$, so that $\epsilon^{-1}\left(x, s \mid w_{0}\right) \rightarrow 0$ as $x \rightarrow \infty$ if and only is $h(x) \rightarrow c$ as $x \rightarrow \infty$.

Now if $h(x)$ is real-valued and $h^{\prime}(x) \neq 0$ an $[0, \infty)$, then $h\left(x_{1}\right) \neq$ $h\left(x_{2}\right)$ for $x_{1} \neq x_{2}$ on this interval, and the limit of $h(x)$ as $x \rightarrow \infty$ exists, finite or infinite, so that in this case (4.9) always has a principal solution, On the other hand, there exist complex-valued $h(x)$ satisfying (4.10), and for which $h(x)$ does not tend to a limit as $x \rightarrow \infty$. Such an example is provided by $h(x)=4(2+\sin x)^{-1}-2 e^{-x}+i \sin ^{3} x$, $0 \leqq x<\infty$. If in the corresponding equation we set $u=u_{1}+i u_{2}$, $v=v_{1}+i v_{2}$ the equivalent system in $u_{1}, u_{2}, v_{1}, v_{2}$ is a system with real coefficients for which the corresponding $2 \times 2$ matrix $\Theta^{-1}\left(x, s \mid W_{0}\right)$ does not tend to a limit as $x \rightarrow \infty$.

5. Self-adjoint systems. Attention will now be restricted to identically normal systems (2.1) which satisfy the self-adjointness conditions (2.21), and also the following hypothesis:

S. $_{0}$. The matrix $B(x)$ is non-negative definite a.e. on $X$.

The condition $\mathfrak{W}_{0}$, with $x$ restricted to a subinterval $[c, d]$, will be denoted by $\mathfrak{S}_{0}[c, d]$.

THEOREM 5.1. If an identically normal system (2.1) satisfying (2.21) and $\hat{\xi}_{0}$ is non-oscillatory on $X_{0}:\left(a_{0}, \infty\right)$, then this system possesses a principal solution at $\infty$. Indeed, if $a_{0}<r<s<t<\infty,\left(U_{s r}(x)\right.$; $\left.V_{s r}(x)\right)$ is the solution of (2.2) satisfying $U_{s r}(r)=0, U_{s r}(s)=E$, and $\left(U_{s t}(s) ; V_{s t}(x)\right)$ is the solution of $(2.2)$ satisfying $U_{s t}(s)=E, U_{s t}(t)=0$, then $V_{s r}(s)>V_{s d}(s)>V_{s t}(s)$ for $a_{0}<r<s<t<d<\infty$, and consequently $V_{s \infty}=\lim _{t \rightarrow \infty} V_{s t}(s)$ exists, and the solution $\left(U_{s \infty}(x) ; V_{s \infty}(x)\right)$ of (2.2) satisfying $U_{s \infty}(s)=E, V_{s \infty}(s)=V_{s \infty}$ is a principal solution at co with $U_{s \infty}(x)$ non-singular on $X_{0}$.

For the case of a system (2.1) arising as the accessory system for a variational problem of Bolza type the result of Theorem 5.1 is given in Reid [9]. For such accessory systems the matrix $B(x)$ is of constant rank a.e. on $X$, whereas for the more general system the rank of $B(x)$ may not be constant a.e. on $X$. In particular, the more general problem includes as a very special instance systems that may be described roughly as arising through the adjunction at interfaces of a sequence of different problems, each of the accessory problem 
type on a corresponding interval.

The above Theorem 5.1 may be established by direct generalizations of the methods used in proving Theorem 5.1 in Reid [9], and this extension is immediate once one has established the results corresponding to Theorems $4.1,4.2$, and 4.3 of [9]. If $[c, d]$ is a compact subinterval of $X$ let $\mathscr{D}[c, d]$ denote the class of pairs of $n$-dimensional vector functions $\eta(x), \zeta(x)$ with $\eta(x)$ a.c. on $[c, d], \zeta(x) \in \mathbb{R}_{\infty}[c, d]$, the class of vector functions Lebesgue measurable and essentially bounded on $[c, d]$, and such that $L_{1}[\eta, \zeta] \equiv \eta^{\prime}-A(x) \eta-B(x) \zeta=0$ a.e. on this interval. The subclass of $\mathscr{D}[c, d]$ on which $\eta(c)=0=\eta(d)$ will be designated by $\mathscr{Q}_{0}[c, d]$. Moreover, let $\mathfrak{Q}[c, d]$ denote the condition that the functional

$$
I[\eta, \zeta ; c, d] \equiv \int_{c}^{a}\left[\zeta^{*}(x) B(x) \zeta(x)+\eta^{*}(x) C(x) \gamma(x)\right] d x
$$

is positive definite on $\mathscr{D}_{0}[c, d]$, that is, $I[\gamma, \zeta ; c, d] \geqq 0$ for $(\eta, \zeta) \in$ $\mathscr{D}_{0}[c, d]$, and the equality sign holds only if $B(x) \zeta(x)=0$ a.e. and $\eta(x) \equiv 0$ on $[c, d]$. The following theorem presents a basic result concerning non-oscillation on a compact interval, and is the result for (2.1) corresponding to Theorem 4.1 of Reid [9].

THEOREM 5.2. If (2.1) is an identically normal system satisfying (2.21) on a compact interval [c,d], then $\mathfrak{5} .[c, d]$ holds if and only if $\mathfrak{g}_{0}[c, d]$ holds, together with one of the following:

(i) (2.1) is non-oscillatory on $[c, d]$;

(ii) there exists a solution $(U(x) ; V(x))$ of $(2.2)$ with $U(x)$ nonsingular on $[c, d]$ and $U^{*}(x) V(x)-V^{*}(x) U(x) \equiv 0$.

For systems (2.1) that arise as accessory systems for variational problems the result of Theorem 5.2 consists of the Legendre or Clebsch condition and a special oscillation theorem in the extension of the classical Sturmian theory to self-adjoint systems as initiated by $M$. Morse; for brief historical statements and references the reader is referred to the author's papers [8; 9] and their bibliographies. If $B(x)$ is positive definite a.e. on $[c, d]$ a proof is contained in Theorem 2.1 of Reid [8], and in the following discussion will be limited to certain aspects that differ from the special cases treated previously.

Theorem 5.2 will be established by proving the following sequence of statements: (a) $\mathfrak{S}_{0}[c, d]$, (ii) $\rightarrow \mathfrak{S}_{2}[c, d]$; (b) $\mathfrak{S}_{[}[c, d] \rightarrow$ (i), $\mathfrak{S}_{0}[c, d]$; (c) $\mathfrak{g}_{0}[c, d]$, (i) $\rightarrow$ (ii).

Statement (a) is an immediate consequence of the relation

$$
\begin{gathered}
I|\gamma, \zeta ; c, d|=\int_{c}^{a}\left(\zeta^{*}-\eta^{*} W\right) B(\zeta-W \eta) d x \geqq 0 \\
\text { for }(\eta, \zeta) \in \mathscr{Z}_{0}[c, d],
\end{gathered}
$$


where $W(x)=V(x) U^{-1}(x)$, since in view of $\mathfrak{g}_{0}[c, d]$ equality in (5.2) holds only if a.e. on $[c, d]$ we have $0=B(\zeta-W \eta)=U\left[U^{-1} \eta\right]^{\prime}$, so that $U^{-1}(x) \eta(x) \equiv U^{-1}(c) \eta(c)=0$, and hence $\eta(x) \equiv 0, B(x) \zeta(x)=0$ a.e. on $[c, d]$. In turn, (5.2) follows from the more general fact that if $U(x)$ and $V(x)$ are $n \times r$ a.c. matrices on $[c, d]$, and for $\alpha=1,2$ the vector functions $\eta_{\alpha}(x)$ are a.c. and $\zeta_{\alpha}(x) \in \mathfrak{R}_{\infty}[c, d]$, while there exist a.c. $r$-dimensional vector functions $h_{\alpha}(x)$ such that $\eta_{\alpha}(x)=U(x) h_{\alpha}(x)$ on $[c, d]$, then we have the identity

$$
\begin{aligned}
\zeta_{1}^{*} B \zeta_{2} & +\eta_{1}^{*} C \eta_{2} \\
= & \left(\zeta_{1}^{*}-h_{1}^{*} V^{*}\right) B\left(\zeta_{2}-V h_{2}\right)-h_{1}^{*} V^{*} L_{1}\left[\eta_{2}, \zeta_{2}\right]-\left(L_{1}\left[\eta_{1}, \zeta_{1}\right]\right)^{*} V h_{2} \\
& -h_{1}^{*}\left\{U^{*} L_{2}[U, V]-V^{*} L_{1}[U, V]\right\} h_{2}-h_{1}^{*}\left[U^{*} V-V^{*} U\right] h_{2}^{\prime} \\
& +\left[h_{1}^{*} U^{*} V h_{2}\right]^{\prime} .
\end{aligned}
$$

For the proof of statement (b), it is to be noted that if $(u, v)$ is a solution of (2.1) with $u(a)=0=u(b)$, where $c \leqq a<b \leqq d$, then for $\eta(x)=u(x), \zeta(x)=v(x)$ on $[a, b]$ and $\eta(x) \equiv 0, \zeta(x) \equiv 0$ elsewhere, we have

$$
I[\eta, \zeta ; c, d]=I[u, v ; a, b]=\left.u^{*} v\right|_{a} ^{b}=0,
$$

so that for general self-adjoint problems (2.1) condition $\mathfrak{L}_{+}[c, d]$ implies (i).

The fact that $\mathfrak{S}_{+}[c, d]$ implies $\mathfrak{S}_{0}[c, d]$ under the general conditions of the theorem may be proved by indirect argument. If it is not true that $B(x) \geqq 0$ a.e. on $[c, d]$, in view of the integrability of $B(x)$ on $[c, d]$, and the separability of finite dimensional Euclidean space, it follows that there exists a constant vector $\zeta_{0}$ with $\left|\zeta_{0}\right|=1$ and positive constants $k_{1}, k_{2}$ such that $X_{0}=\left\{x|c \leqq x \leqq d| B,(x) \mid \leqq k_{1}\right.$, $\left.\zeta_{0}^{*} B(x) \zeta_{0}<-k_{2}\right\}$ is of positive measure. If $Y(x)$ is a fundamental matrix of $Y^{\prime}=A(x) Y$, and $k_{3}$ a constant such that $\left|Y(x) Y^{-1}(t)\right| \leqq k_{3}$ for $x$ and $t$ on $[c, d]$, let $s$ be a point of outer density of $X_{0}$ belonging to $(c, d)$, and choose $a, b$ such that $c<a<s<b \leqq d$, and

$$
(b-a)^{-1}>\left(k_{1}^{2} k_{3}^{2} / k_{2}\right) \int_{c}^{a}|C(x)| d x .
$$

If $e(x)$ denotes the characteristic function of $X_{0}$, then there exists a continuous scalar function $g(x) \neq \equiv$ on $[a, b]$, and such that the solution $y(x)$ of $L_{1}\left[y, \zeta_{0} e g\right]=0, \quad y(a)=0$, satisfies $y(b)=0$ and $y(x) \neq 0$ on $[a, b]$, indeed, $g(x)$ may be chosen of the form $g(x)=c_{0}+c_{1} x+\cdots+$ $c_{n} x^{n}$ with $\left|c_{0}\right|^{2}+\cdots+\left|c_{n}\right|^{2}=1$. For $\zeta_{1}(x)=\zeta_{0} e(x) g(x)$ we have $y(x)=$ $\int_{a}^{x} Y(x) Y^{-1}(t) B(t) \zeta_{1}(t) d t$, and in view of the definitive properties of $k_{1}$ and $k_{3}$ we have $|y(x)| \leqq k_{1} k_{3} \int_{a}^{b}|e(x) g(x)| d x$ for $a \leqq x \leqq b$. If $\eta(x)=$ $y(x), \zeta(x)=\zeta_{1}(x)$ on $[a, b]$, and $\eta(x) \equiv 0, \zeta(x) \equiv 0$ on $[c, a]$ and $[b, d]$, 
then $(\eta(x), \zeta(x)) \in \mathscr{Q}[c, d]$ and

$$
\begin{aligned}
I[\eta, \zeta ; c, d] \leqq & -k_{2} \int_{a}^{b}|e(x) g(x)|^{2} d x \\
& +k_{1}^{i} k_{s}^{2}\left(\int_{a}^{b}|e(x) g(x)| d x\right)^{2}\left(\int_{c}^{a}|C(x)| d x\right) .
\end{aligned}
$$

As $\left(\int_{a}^{b}|e(x) g(x)| d x\right)^{2} \leqq(b-a) \int_{a}^{b}|e(x) g(x)|^{2} d x$ by the Schwarz inequality, and $\int_{a}^{b}|e(x) g(x)|^{2} d x>0$, with the aid of (5.4) it then follows that

$$
I[\eta, \zeta ; c, d] \leqq-\left(k_{2}-(b-a) k_{i}^{2} k_{j}^{2} \int_{c}^{a}|C(x)| d x\right) \int_{a}^{b}|e(x) g(x)|^{2} d x<0,
$$

sont $\mathfrak{a}_{1} \mathrm{y}$ to the condition $\mathfrak{S}_{+}[c, d]$.

The above statement (c) may be proved by exactly the same type of argument as that used to establish the statement (f) for the proof of Theorem 2.1 in Reid [3], with the functional (5.2) replacing the $I[\eta]$ of [s], and details will be omitted here.

It is to be remarked that the result of Theorem 5.2 is true without the assumption of identical normality; indeed, the above proofs of statements (a) and (b) do not use this condition, and (c) may be established without this hypothesis by using methods that have been employed for the special systems arising as accessory systems for Bolza problems, (see Bliss [1, §89]).

With Theorem 5.2 thus established, for the general system under consideration one may prove the results corresponding to Theorems 4.2 and 4.3 of Reid [9], and then proceed as in [9] to obtain the result of Theorem 5.1. The proofs of this section are distinctly variational in character, and are in essence "classical variational proofs phrased in terms of canonical variables." For example, for accessory systems of Bolza type variational problems the identity (5.3) is in essence the well-known Clebsch transformation of the second variation, (see Bliss [1, §23,39], and for such systems the fact that $\mathfrak{L}_{+}[c, d]$ implies $s_{0}[c, d]$ is the "Legendre" or "Clebsch" condition.

In passing, it is to be commented that for a system (2.1) satisfying (2.21) and identically normal on a compact interval $[c, d]$ one may obtain the full extension of Theorem 2.1 of Reid [8], as well as the corresponding criteria $i v_{R}$ and $v_{h}$, (see [8, p. 741]), of that paper. In particular, if $U(x)$ and $V(x)$ are $n \times n$ matrices a.c. on $[c, d]$, and we set

$$
\Lambda[U, V]=U^{*}(x) L_{2}[U, V]-V^{*}(x) L_{1}[U, V],
$$

then $\Lambda[U, V]-(\bigwedge[U, V])^{*} \equiv\left(U^{*} V-V^{*} U\right)^{\prime}$; moreover, whenever $U(x)$ is non-singular on $[c, d]$ the matrix $W(x)=V(x) U^{-1}(x)$ is such 
that

$$
\begin{gathered}
\Lambda[U, V]=U^{*} K[W] U+U^{*}\left(W-W^{*}\right) L_{1}[U, V], \\
U^{*} V-V^{*} U=U^{*}\left(W-W^{*}\right) U .
\end{gathered}
$$

Consequently, corresponding to the statement of $[8, p .741]$ on the condition $v_{R}$ we have: for an identically normal system (2.1) satisfying (2.21) on $[c, d]$ condition $\mathfrak{L}_{+}[c, d]$ holds if and only if $\mathfrak{E}_{0}[c, d]$ holds and there is an $n \times n$ hermitian a.c. matrix $W(x)$ such that a.e. on $[c, d]$ the hermitian matrix $K[W]$ is non-positive definite.

THeOREM 5.3. If (2.1) is an identically normal system satisfying (2.21) and $\mathfrak{F}_{0}$, and which is non-oscillatory on an interval $X:(a, \infty)$, then:

(a) If $W_{0}(x)$ is an hermitian solution of (2.6) on a subinterval $[s, \infty)$ of $X$, and $W(x)$ is the solution of (2.6) satisfying $W(s)=$ $W_{0}(s)+\Gamma$, then $W(x)$ exists on $[s, \infty)$ if either $\Gamma_{\Im}$ is definite, or if there are real constants $\lambda_{0}>0, \lambda_{1}$ such that $\lambda_{0} \Gamma_{\Re}+\lambda_{1} \Gamma_{\Im} \geqq 0$; in particular, if $\Gamma$ is an hermitian matrix satisfying $\Gamma \geqq 0$ then $W(x)-W_{0}(x) \geqq 0$ on $[s, \infty)$.

(b) If $W_{\infty}(x)$ is the distinguished solution of (2.6) at $\infty$, then $W_{\infty}(x)$ exists and is hermitian on $X$; moreover if $s \in X$ and $W(x)$ is a solution of (2.6) satisfying $W(s)=W_{\infty}(s)+\Gamma$, where $\Gamma$ is an hermitian matrix that fails to be non-negative, then $W(x)$ does not exist throughout $[s, \infty)$.

For a system (2.1) satisfying (2.21) it follows that $G\left(x, s \mid W_{0}\right) \equiv$ $H^{*}\left(x, s \mid W_{0}\right)$ for an hermitian solution $W_{0}(x)$ of (2.6), and for such a system which is non-oscillatory and satisfies $\mathscr{S}_{0}$ on $X$ we have that $\Theta\left(x, s \mid W_{0}\right)>0$ for $x \in X_{s}^{+}$. If $W(x)$ is a solution of (2.6) satisfying $W(s)=W_{0}(s)+\Gamma$, then Lemma 2.1 implies that $W(x)$ exists on $[s, \infty)$ if and only if $E+\Theta\left(x, s \mid W_{0}\right) \Gamma$ is non-singular on $[s, \infty)$, and this latter condition is equivalent to the non-singularity of $\Theta^{-1}\left(x, s \mid W_{0}\right)+\Gamma$ on $(s, \infty)$. If $x \in[s, \infty)$ and $\left[\Theta^{-1}\left(x, s \mid W_{0}\right)+\Gamma\right] \eta=0$, then

$$
\eta^{*}\left[\Theta^{-1}\left(x, s \mid W_{0}\right)+\Gamma_{\Re}\right] \eta=-i \eta * \Gamma_{\Im} \eta,
$$

and hence

$$
\eta^{*}\left[\Theta^{-1}\left(x, s \mid W_{0}\right)+\Gamma_{\Re}\right] \eta=0, \quad \eta^{*} \Gamma_{\Im} \eta=0 .
$$

Now if $\Gamma_{\Im}$ is definite the second condition of (5.5) implies $\eta=0$; on the other hand, if $\lambda_{0}>0, \lambda_{1}$ are real constants such that $\lambda_{0} \Gamma_{\Re}+\lambda_{1} \Gamma_{\Im} \geqq$ 0 , then from (5.5) it follows that $\eta^{*} \Theta^{-1}\left(x, s \mid W_{0}\right) \eta=0$ and hence $\eta=0$. Thus $\Theta^{-1}\left(x, s \mid W_{0}\right)+\Gamma$ is non-singular on $(s, \infty)$ and $W(x)$ exists on 
$[s, \infty)$. In particular, if $\Gamma$ is an hermitian matrix satisfying $\Gamma \geqq 0$ then this latter criterion implies that $W(x)$ exists on $[s, \infty)$, and the conclusion $W(x)-W_{0}(x) \geqq 0$ follows from the representation formula (2.11), and the fact that if matrices $\Theta, \Gamma$ are such that $\Gamma \geqq 0, \Theta>0$, and $E+\theta \Gamma$ is non-singular, then

$$
\Gamma[E+\theta \Gamma]^{-1} \equiv[E+\Theta \Gamma]^{*-1}[\Gamma+\Gamma \Theta \Gamma][E+\Theta \Gamma]^{-1} \geqq 0 .
$$

In view of Theorems 4.2 and 5.1 , if $\left(U_{\infty}(x) ; V_{\infty}(x)\right)$ is a principal solution of (2.2) at $\infty$ then $U_{\infty}^{*} V_{\infty}-V_{\infty}^{*} U_{\infty} \equiv 0$ and $U_{\infty}(x)$ is non-singular on $X:(a, \infty)$, so that the corresponding distinguished solution $W_{\infty}(x)=$ $V_{\infty}(x) U_{\infty}^{-1}(x)$ of (2.6) is hermitian and exists on $X$. Consequently, if $s \in X$ then $\theta\left(x, s \mid W_{\infty}\right)>0$ for $x \in(s, \infty)$, and hence $\Theta^{-1}\left(x, s \mid W_{\infty}\right)+\Gamma$ is hermitian on $(s, \infty)$ for $\Gamma$ an hermitian matrix. Moreover, since $W_{\infty}(x)$ is the distinguished solution of (2.6) at $\infty, \Theta^{-1}\left(x, s \mid W_{\infty}\right)+\Gamma \rightarrow$ $\Gamma$ as $x \rightarrow \infty$, while $\Theta^{-1}\left(x, s \mid W_{\infty}\right)+\Gamma$ is positive definite for $x>s$ and sufficiently close to $s$. Consequently, if $\Gamma$ fails to be non-negative definite there exists a value $t \in(s, \infty)$ such that $\Theta^{-1}\left(t, s \mid W_{\infty}\right)+\Gamma$ is singular, so that $W_{\infty}(x)$ is not extensible to an interval containing $t$, in contradiction to the existence of $W_{\infty}(x)$ on $X$.

Combining the conclusions (a) and (b) we have that if the distinguished solution $W_{\infty}(x)$ of $(2.6)$ exists on an interval $(a, \infty)$ then an hermitian solution $W(x)$ of $(2.6)$ exists on a subinterval $[s, \infty)$ of $(\alpha, \infty)$ if and only if $W(x)-W_{\infty}(x) \geqq 0$ for at least one value, and consequently all values $\}$, on $[s, \infty)$. For the case of systems (2.1) with real coefficients satisfying (2.21), and for which $B(x)>0$ on $X$, this result has been proved by Sandor [11]; due to this property he has designated as "the right-hand frontier solution" the solution of (2.6) that we have called the distinguished solution at $\infty$.

6. Systems non-oscillatory on intervals $(-\infty, a)$ and $(-\infty, \infty)$. The behavior of (2.2) and (2.6) on an interval $(-\infty, a)$ is obviously equivalent under the reflective transformations $U^{0}(x)=U(-x), V^{0}(x)=$ $V(-x), W^{0}(x)=W(-x)$ to the behavior of the respective equations

$$
\begin{aligned}
& U^{0^{\prime}}=-A(-x) U^{0}-B(-x) V^{0}, V^{0^{\prime}}=-C(-x) U^{0}+D(-x) V^{0}, \\
& W^{0^{\prime}}-W^{0} A(-x)-D(-x) W^{0}-W^{0} B(-x) W^{0}+C(-x)=0,
\end{aligned}
$$

on $(-a, \infty)$. A principal solution of $(2.2)$ at $-\infty$, and the associated distinguished solution $W_{-\infty}(x)$ of (2.6) at $-\infty$, are defined as the images under the above transformations of a principal solution of $\left(2.2^{\circ}\right)$ at $\infty$ and the associated distinguished solution $W_{\infty}^{0}(x)$ of $\left(2.6^{\circ}\right)$ at $\infty$. The analogues of Theorems 4.2, 5.1 and 5.3 for intervals $X:(-\infty, a)$ are immediate, and will not be presented in any further 
detail, with the exception of the following results, which are consequences of combined results of these theorems for (2.2), (2.6) and $\left(2.2^{\circ}\right),\left(2.6^{0}\right)$. For the systems considered by Sandor, results equivalent to those of Theorem 6.1 are given in $[11, \S 7]$.

THEOREM 6.1. If on the real line $(-\infty, \infty)$ the system (2.1) is identically normal, satisfies (2.21) and $\mathfrak{K}_{0}$, and is non-oscillatory, then the distinguished solutions $W_{\infty}(x)$ and $W_{-\infty}(x)$ of (2.6) are individually hermitian on $(-\infty, \infty)$ and such that:

(a) If $\left(U_{t}(x) ; V_{t}(x)\right)$ is the solution of (2.2) determined by (4.2) for $-\infty<t<\infty$; and $W_{t}(x)=V_{t}(x) U_{t}^{-1}(x)$, then $W_{t}(x) \rightarrow W_{\infty}(x)$ as $t \rightarrow \infty$, and $W_{t}(x) \rightarrow W_{-\infty}(x)$ as $t \rightarrow-\infty$.

(b) If $W(x)$ is an hermitian solution of (2.6) which exists on $(-\infty, \infty)$ then $W(x)-W_{\infty}(x) \geqq 0$ and $W_{-\infty}(x)-W(x) \geqq 0$ throughout $(-\infty, \infty)$, while if $W(x)$ is an hermitian solution of (2.6) for which at some value $s$ the matrix $W(s)-W_{\infty}(s),\left\{W_{-\infty}(s)-W(s)\right\}$, fails to be nonnegative definite then $W(x)$ does not exist throughout the interval $[s, \infty),\{(-\infty, s]\}$.

For example, the scalar system

$$
u^{\prime}=v, \quad v^{\prime}=u
$$

is non-oscillatory on $(-\infty, \infty)$, and $u_{t}(x)=\sinh (x-t), \quad v_{t}(x)=$ $\cosh (x-t)$. The corresponding Riccati equation (2.6) is

$$
w^{\prime}+w^{2}-1=0,
$$

with respective solutions $w_{t}(x)=\operatorname{coth}(x-t), w_{\infty}(x) \equiv-1$, and $w_{-\infty}(x) \equiv 1$.

7. Systems with constant coefficients. If the coefficient matrices $A, B, C, D$ are constant, and $(U(x) ; V(x))$ is a solution of (2.2), then $(U(x-c) ; V(x-c))$ is also a solution for arbitrary real values $c$. Consequently, (2.1) is non-oscillatory on an interval $(a, \infty)$ or $(-\infty, a)$ if and only if it is non-oscillatory on the whole infinite line $(-\infty, \infty)$. Moreover, if $\left(U_{t}(x) ; V_{t}(x)\right)$ is the solution of (2.2) satisfying (4.2) then $U_{s}(x) \equiv U_{t}(x-s+t), \quad V_{s}(x) \equiv V_{t}(x-s+t)$, and the corresponding solution $W_{t}(x)=V_{t}(x) U_{t}^{-1}(x)$ of $(2.6)$ exists on an interval [c,d] if and only if $W_{s}(s)=V_{s}(x) U_{s}^{-1}(x)$ exists on $[c+s-t, d+s-t]$. For systems with constant coefficients the following result is a consequence of Theorem 4.2.

THEOREM 7.1. A system (2.2) with constant coefficients, and which is identically normal and non-oscillatory on $(-\infty, \infty)$, has a principal solution at $\infty\{a t-\infty\}$ if and only if the solution $\left(U_{0}(x) ; V_{0}(x)\right)$ of (2.2) for which $U_{0}(0)=0, V_{0}(0)=E$ is such that $W_{0}(x)=V_{0}(x) U_{0}^{-1}(x)$ 
converges to a limit $W_{\infty}\left\{W_{-\infty}\right\}$ as $x \rightarrow-\infty\{x \rightarrow \infty\}$; the corresponding distinguished solution of (2.6) at $\infty \quad\{a t-\infty\}$ is $W_{\infty}(x) \equiv W_{\infty}$ $\left\{W_{-\infty}(x) \equiv W_{-\infty}\right\}$.

In turn, Theorems 5.1 and 6.1 imply the following results for systems with constant coefficients.

THEOREM 7.2. A system (2.2) with constant coefficient matrices satisfying $A^{*}=D, C^{*}=C, B^{*}=B \geqq 0$, and which is identically normal, is non-oscillatory on $(-\infty, \infty)$ if and only if there exists an hermitian constant matrix $W$ satisfying the algebraic matrix equation

$$
W A+A^{*} W+W B W-C=0 ;
$$

moreover, if such a system is non-oscillatory on $(-\infty, \infty)$ then there exist hermitian matrices $W_{\infty}$ and $W_{-\infty}$ which are individually solutions of (7.1), and are extreme solutions for (2.6) in the sense that if $W(x)$ is any hermitian solution of $(2.6)$ on $(-\infty, \infty)$ then $W_{\infty} \leqq$ $W(x) \leqq W_{-\infty}$; in particular, if $W$ is any hermitian solution of (7.1) then $W_{\infty} \leqq W \leqq W_{-\infty}$.

In particular, if $B$ and $C$ are constant matrices the system

$$
u^{\prime}=B v, \quad v^{\prime}=C u,
$$

is identically normal on $(-\infty, \infty)$ if and only if $B$ is non-singular, and the following result is an immediate consequence of the above theorem.

CoRollary. If $B$ and $C$ are constant hermitian matrices with $B>0$, then (7.2) is non-oscillatory on $(-\infty, \infty)$ if and only if $C \geqq 0$, and whenever this latter condition holds then

$$
W_{\infty}=-B^{-1 / 2}\left[B^{1 / 2} C B^{1 / 2}\right]^{1 / 2} B^{-1 / 2}
$$

and $W_{-\infty}=-W_{\infty}$.

It is to be remarked that this corollary provides a differential equation algorism for the nonnegative definite square root of a given nonnegative definite matrix $C$ :

$$
C^{1 / 2}=\lim _{x \rightarrow \infty} V_{0}(x) U_{0}^{-1}(x)=-\lim _{x \rightarrow-\infty} V_{0}(x) U_{0}^{-1}(x),
$$

where $\left(U_{0}(x) ; V_{0}(x)\right)$ is the solution of $U^{\prime}=V, V^{\prime}=C U$ satisfying $U(0)=0, \quad V(0)=E$. 


\section{BIBLIOGRAPHY}

1. G. A. Bliss, Lectures on the calculus of variations, University of Chicago Press, 1946.

2. P. Hartman and A. Wintner, On the assignment of asymptotic values for the solutions of linear differential equations of the second order, Amer. J. Math., 77 (1955), 475-483.

3. P. Hartman, Self-adjoint, non-oscillatory systems of ordinary, second order linear differential equations, Duke Math. J., 24 (1957), 25-35.

4. W. Leighton and M. Morse, Singular quadratic functionals, Trans. Amer. Math. Soc., 40 (1936), 252-286.

5. W. Leighton, Principal quadratic functionals, Trans. Amer. Math. Soc., 67 (1949), 253-274.

6. J. J. Levin, On the matrix Riccati equation, Proc. Amer. Math. Soc., 10 (1959), 519-524.

7. W. T. Reid, A matrix differential equation of Riccati type, Amer. J. Math., 68 (1946), 237-246; Addendum, ibid, 70 (1948), 460.

8. - Oscillation criteria for linear differential systems with complex coefficients, Pacific J. Math., 6 (1956), 733-751.

9. - Principal solutions of non-oscillatory self-adjoint linear differential systems, Pacific J. Math., 8 (1958), 147-169.

10. Properties of solutions of a Riccati matrix differential equation, J. of Math. and Mech., 9 (1960), 749-770.

11. S. Sandor, Sur l'équation différentielle matricielle de type Riccati, Bull. Math. Soc. Sci. Math. Phys., R. P. Roumaine (N.S.) 3 (51), 1959, 229-249.

12. W. M. Whyburn, Matrix differential equations, Amer. J. Math., 56 (1934), 587-592.

STATE UNIVERSiTY OF IOWA 



\title{
PACIFIC JOURNAL OF MATHEMATICS
}

\author{
EDITORS
}

\author{
Ralph S. Phillips \\ Stanford University \\ Stanford, California \\ M. G. Arsove \\ University of Washington \\ Seattle 5 , Washington
}

\author{
J. DugunduI \\ University of Southern California \\ Los Angeles 7, California
}

Lowell J. Paige

University of California

Los Angeles 24, California

\section{ASSOCIATE EDITORS}

E. F. BECKENBACH

T. M. CHERRY
D. DERRY
M. OHTSUKA

H. L. ROYDEN

E. SPANIER
E. G. STRAUS

F. WOLF

\section{SUPPORTING INSTITUTIONS}

UNIVERSITY OF BRITISH COLUMBIA CALIFORNIA INSTITUTE OF TECHNOLOGY UNIVERSITY OF CALIFORNIA MONTANA STATE UNIVERSITY UNIVERSITY OF NEVADA NEW MEXICO STATE UNIVERSITY OREGON STATE UNIVERSITY UNIVERSITY OF OREGON OSAKA UNIVERSITY UNIVERSITY OF SOUTHERN CALIFORNIA
STANFORD UNIVERSITY

UNIVERSITY OF TOKYO

UNIVERSITY OF UTAH

WASHINGTON STATE UNIVERSITY

UNIVERSITY OF WASHINGTON

AMERICAN MATHEMATICAL SOCIETY CALIFORNIA RESEARCH CORPORATION SPACE TECHNOLOGY LABORATORIES NAVAL ORDNANCE TEST STATION

Mathematical papers intended for publication in the Pacific Journal of Mathematics should be typewritten (double spaced), and the author should keep a complete copy. Manuscripts may be sent to any one of the four editors. All other communications to the editors should be addressed to the managing editor, L. J. Paige at the University of California, Los Angeles 24, California.

50 reprints per author of each article are furnished free of charge; additional copies may be obtained at cost in multiples of 50 .

The Pacific Journal of Mathematics is published quarterly, in March, June, September, and December. Effective with Volume 13 the price per volume (4 numbers) is $\$ 18.00$; single issues, $\$ 5.00$. Special price for current issues to individual faculty members of supporting institutions and to individual members of the American Mathematical Society: $\$ 8.00$ per volume; single issues \$2.50. Back numbers are available.

Subscriptions, orders for back numbers, and changes of address should be sent to Pacific Journal of Mathematics, 103 Highland Boulevard, Berkeley 8, California.

Printed at Kokusai Bunken Insatsusha (International Academic Printing Co., Ltd.), No. 6, 2-chome, Fujimi-cho, Chiyoda-ku, Tokyo, Japan.

PUBLISHED BY PACIFIC JOURNAL OF MATHEMATICS, A NON-PROFIT CORPORATION

The Supporting Institutions listed above contribute to the cost of publication of this Journal, but they are not owners or publishers and have no responsibility for its content or policies. 


\section{Pacific Journal of Mathematics}

\section{Vol. 13, No. 2 \\ April, 1963}

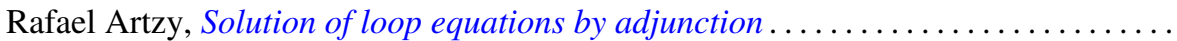

Earl Robert Berkson, A characterization of scalar type operators on reflexive

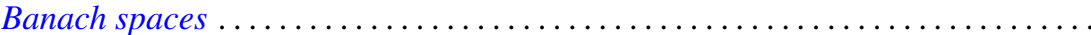

Mario Borelli, Divisorial varieties

365

Raj Chandra Bose, Strongly regular graphs, partial geometries and partially

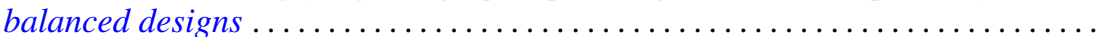

389

R. H. Bruck, Finite nets. II. Uniqueness and imbedding ............... 421

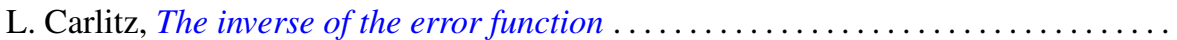

Robert Wayne Carroll, Some degenerate Cauchy problems with operator

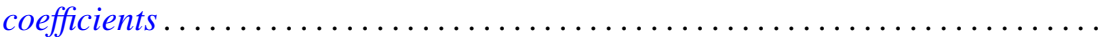

Michael P. Drazin and Emilie Virginia Haynsworth, A theorem on matrices of 0 's

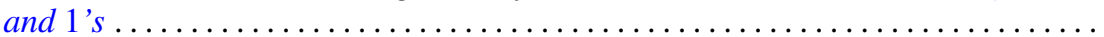

Lawrence Carl Eggan and Eugene A. Maier, On complex approximation .......... James Michael Gardner Fell, Weak containment and Kronecker products of group

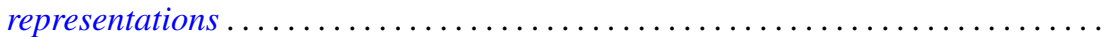

Paul Chase Fife, Schauder estimates under incomplete Hölder continuity

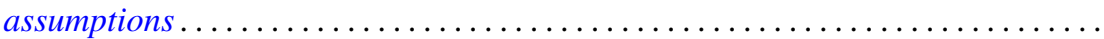

Shaul Foguel, Powers of a contraction in Hilbert space ...................

Neal Eugene Foland, The structure of the orbits and their limit sets in continuous

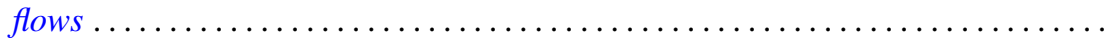

Frank John Forelli, Jr., Analytic measures . . . . . . . . . . . . . . . . . . . . . 563

Robert William Gilmer, Jr., On a classical theorem of Noether in ideal theory ....... P. R. Halmos and Jack E. McLaughlin, Partial isometries .

Albert Emerson Hurd, Maximum modulus algebras and local approximation in

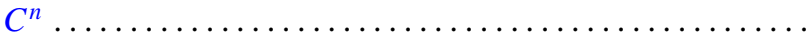

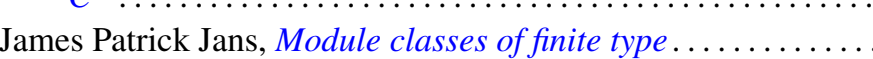

Betty Kvarda, On densities of sets of lattice points ...

H. Larcher, A geometric characterization for a class of discontinuous groups of linear fractional transformations .

John W. Moon and Leo Moser, Simple paths on polyhedra .

T. S. Motzkin and Ernst Gabor Straus, Representation of a point of a set as sum of

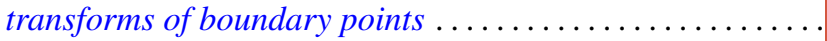

Rajakularaman Ponnuswami Pakshirajan, An analogue of Kolmogorov's three-series theorem for abstract random variables ...............

Robert Ralph Phelps, Čebyšev subspaces of finite codimension in $C(X)$...

James Dolan Reid, On subgroups of an Abelian group maximal disjoint from a given subgroup ...

William T. Reid, Riccati matrix differential equations and non-oscillation criteria for associated linear differential systems .................

Georg Johann Rieger, Some theorems on prime ideals in algebraic number fields ...

Gene Fuerst Rose and Joseph Silbert Ullian, Approximations of functions on the

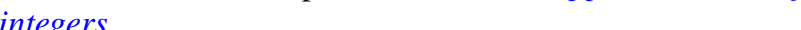

F. J. Sansone, Combinatorial functions and regressive isols . . . . . . . . . . 703

Leo Sario, On locally meromorphic functions with single-valued moduli . . . . . . . 709

Takayuki Tamura, Semigroups and their subsemigroup lattices.

Pui-kei Wong, Existence and asymptotic behavior of proper solutions of a class of second-order nonlinear differential equations . . ........... 